\title{
On the convergence difference sequences and the related operator norms
}

\author{
P. Baliarsingh \\ Department of Mathematics, Gangadhar \\ Meher University, Sambalpur-768004, \\ Odisha, India \\ email: pb.math10@gmail.com
}

\author{
L. Nayak* \\ Department of Mathematics, School \\ of Applied Sciences, Kalinga \\ Institute of Industrial Technology, \\ Bhubaneswar-751024, India \\ email: laxmipriyamath@gmail.com
}

\author{
S. Samantaray \\ Department of Mathematics, School of Applied \\ Sciences, Kalinga Institute of Industrial Technology, \\ Bhubaneswar-751024, India \\ Kalinga Institute of Industrial Techonology, \\ Bhubaneswar-751024, India \\ email: samantaraysnigdha1995@gmail.com
}

\begin{abstract}
In this note, we discuss the definitions of the difference sequences defined earlier by Kızmaz (1981), Et and Çolak (1995), Malkowsky et al. (2007), Başar(2012), Baliarsingh (2013, 2015) and many others. Several authors have defined the difference sequence spaces and studied their various properties. It is quite natural to analyze the convergence of the corresponding sequences. As a part of this work, a convergence analysis of difference sequence of fractional order defined earlier is presented. It is demonstrated that the convergence of the fractional difference sequence is dynamic in nature and some of the results involved are also inconsistent. We provide certain stronger conditions on the primary sequence and the results due to earlier authors are substantially modified. Some illustrative examples are provided for each point of the modifications. Results on certain operator norms related to the difference operator of fractional order are also determined.
\end{abstract}

2010 Mathematics Subject Classification: 40A07; 40C09; 46B08

Key words and phrases: sequence spaces, difference sequence spaces, difference operators $\Delta^{\alpha}$ and $\Delta^{\mathrm{m}}$, operator norms

${ }^{*}$ corresponding author 


\section{Introduction}

Recently, one of the most interesting areas of research in Mathematics is the study of difference operators and related sequence spaces which has been attracted in different areas of Mathematical sciences especially in applied and computational fields involving calculus, matrix and approximation theory. The idea of difference sequence spaces plays a key role in most of the scientific problems involving the spectral properties of bounded linear operators(see $[2,7,11,15,16,28,29,30]$ ), related topological structures (see $[3,4,19,20,22,26,27])$, matrix transformations(see [5, 12, 18, 19, 21, 23]), compact operators (see $[1,14,24,25])$, fractional calculus $[8,9,10]$, etc.

In fact, the study of all the ideas discussed earlier is only feasible and even possible if the related sequences are convergent.

Let $x=\left(x_{k}\right)$ be any sequence in $w$, the family of all real valued sequences. Let $\mathbb{N}$ be the set of all positive integers and $\mathbb{N}_{0}=\mathbb{N} \cup\{0\}$. A sequence $x=\left(x_{k}\right)$ is said to be of order $k^{\alpha}$, i.e., $x_{k}=\mathcal{O}\left(k^{\alpha}\right)$ if for a positive constant $\mathcal{C}$, we can write

$$
\left|x_{k}\right| \leq \mathcal{C} k^{\alpha}, k=0,1,2,3, \ldots
$$

By $\ell_{\infty}, c$ and $c_{0}$, we denote the spaces of all bounded, convergent and null sequences, respectively, normed by

$$
\|x\|_{\infty}=\sup _{k}\left|x_{k}\right| .
$$

We use the notation $\ell_{p},(1 \leq p<\infty)$ for the space of all $p$-summable sequence with the norm

$$
\|x\|_{p}=\left(\sum_{k=0}^{\infty}\left|x_{k}\right|^{p}\right)^{1 / p} .
$$

The 1st order difference sequence space $X(\Delta)$ for $X \in\left\{\ell_{\infty}, c, c_{0}\right\}$ was introduced by Kizmaz [20] using forward difference operator $\Delta$, where

$$
\Delta x_{k}=x_{k}-x_{k+1},\left(k \in \mathbb{N}_{0}\right) \text {. }
$$

Later on, this idea has been generalized to the case of difference sequence spaces of integer order $\mathrm{m}$ by Et and Çolak [17] using the operator $\Delta^{\mathrm{m}}$ and

$$
\Delta^{m} x_{k}=\sum_{i=0}^{m}(-1)^{i}\left(\begin{array}{c}
m \\
i
\end{array}\right) x_{k+i}, \quad\left(k \in \mathbb{N}_{0}\right) \text {. }
$$


Using Euler gamma function for a proper fraction $\alpha$, the fractional difference sequence $\Delta^{\alpha} x$ of order $\alpha$ was defined by Baliarsingh [4](see also [5, 6]) as

$$
\Delta^{\alpha} \chi_{k}=\sum_{i=0}^{\infty}(-1)^{i} \frac{\Gamma(\alpha+1)}{i ! \Gamma(\alpha-i+1)} x_{k+i},\left(k \in \mathbb{N}_{0}\right) .
$$

By taking inverse transform $\Delta^{-\alpha}$ on the sequence $x=\left(x_{k}\right)$, we write the Eqn. (3) as

$$
\Delta^{-\alpha} x_{k}=x_{k}+\alpha x_{k+1}+\frac{\alpha(\alpha+1)}{2 !} x_{k+2}+\frac{\alpha(\alpha+1)(\alpha+1)}{3 !} x_{k+3}+\ldots
$$

An infinite series has no meaning unless it converges. It is important to mention that in the previous papers, the convergence of the fractional difference sequence defined by (3) and (4) have been presumed without taking any further investigations. Now, in particular, we illustrate the following examples regarding the convergence of these series:

Example 1 Let $\alpha$ be a proper fraction and $x=\left(x_{k}\right)$ be the convergent sequence defined by $\mathrm{x}_{\mathrm{k}}=\frac{1}{3 \mathrm{k}}$ for all $\mathrm{k} \in \mathbb{N}_{0}$. Then, we can easily calculate

$$
\Delta^{\alpha} x_{k}=\sum_{i=0}^{\infty}(-1)^{i} \frac{\Gamma(\alpha+1)}{i ! \Gamma(\alpha-i+1)} \frac{1}{3^{k+i}}=\frac{1}{3^{k}}\left(\frac{2}{3}\right)^{\alpha}=\frac{2^{\alpha}}{3^{k+\alpha}} \rightarrow 0 \text { as } k \rightarrow \infty,
$$

and

$\Delta^{-\alpha} x_{k}=\sum_{i=0}^{\infty} \frac{\alpha(\alpha+1) \ldots(\alpha+i-1)}{\Gamma(i+1)} \frac{1}{3^{k+i}}=\frac{1}{3^{k}}\left(\frac{2}{3}\right)^{-\alpha}=\frac{3^{\alpha-k}}{2^{\alpha}} \rightarrow 0$ as $k \rightarrow \infty$.

Example 2 Let $\mathrm{x}=\left(\mathrm{x}_{\mathrm{k}}\right)$ be the constant sequence with $\mathrm{x}_{\mathrm{k}}=1$ for all $\mathrm{k} \in \mathbb{N}_{0}$. Although the sequence $\mathrm{x}=\left(\mathrm{x}_{\mathrm{k}}\right)$ is convergent, but for a proper fraction $\alpha$, $\Delta^{\alpha} \chi_{k} \rightarrow 0$ as $\mathrm{k} \rightarrow \infty$ whereas, $\Delta^{-\alpha} \chi_{k} \rightarrow \infty$ as $\mathrm{k} \rightarrow \infty$.

Example 3 Let $\mathrm{x}=\left(\mathrm{x}_{\mathrm{k}}\right)$ be the oscillating sequence, defined by $x_{\mathrm{k}}=(-1)^{\mathrm{k}}$ for all $\mathrm{k} \in \mathbb{N}_{0}$. Clearly, the sequence $\mathrm{x}=\left(\mathrm{x}_{\mathrm{k}}\right)$ is divergent and for a proper fraction $\alpha$, we have

$$
\Delta^{\alpha} x_{k}= \begin{cases}2^{\alpha}, & (k \text { is even }) \\ -2^{\alpha}, & (k \text { is odd }),\end{cases}
$$

and

$$
\Delta^{-\alpha} x_{k}= \begin{cases}2^{-\alpha}, & (k \text { is even }) \\ -2^{-\alpha}, & (k \text { is odd })\end{cases}
$$

are also divergent. 
Example 4 Let $\mathrm{x}=\left(\mathrm{x}_{\mathrm{k}}\right)$ be the divergent sequence defined by $\mathrm{x}_{\mathrm{k}}=\mathrm{k}$ for all $\mathrm{k} \in \mathbb{N}_{0}$. Although the sequence $\mathrm{x}=\left(\mathrm{x}_{\mathrm{k}}\right)$ is divergent, but for an integer $\alpha>1$, $\Delta^{\alpha} \chi_{k} \rightarrow 0$ as $\mathrm{k} \rightarrow \infty$ whereas, $\Delta^{-\alpha} \chi_{k} \rightarrow \infty$ as $\mathrm{k} \rightarrow \infty$. For a proper fraction $\alpha$, both of the difference sequences go to $\infty$ as $\mathrm{k} \rightarrow \infty$.

It is remarked that the infinite series defined in (3) and (4) need not be convergent for any arbitrary sequence $x=\left(x_{k}\right)$ and any proper fraction $\alpha$. Therefore, it is quite difficult to study and analyze the behaviors of the related sequence spaces for fractional cases. As the convergence of the difference sequence $\Delta^{\alpha} x$ depends on the nature and behavior of the sequence $x$ and the value $\alpha$, it has been observed that the properties such as linearity and exponent rules of the difference operator $\Delta^{\alpha}$ are violating in certain particular cases. As a consequence of these violations, it is concluded that Theorems 1, 2 and 3 due to $[4,5]$ are not stable and need certain additional conditions in order to provide their substantial modifications.

The primary objective of this note is to study the convergence of the fractional difference sequences, the dynamic nature of the fractional difference operator $\Delta^{\alpha}$ in detail and apply the same to modify Theorems 1, 2 and 3 of $[4,5]$. Now, we analyze the convergence of the difference sequence $\Delta^{\alpha} \chi$ for different choice of $\alpha$ in detail, (i.e., $\alpha>0, \alpha<0$ and $\alpha \in \mathbb{N}$ ) by using the following theorems.

\section{Main results}

Theorem 1 The series defined in (3) is convergent for any $\alpha=\mathrm{n} \in \mathbb{N}$ if the sequence $\mathrm{x}=\left(\mathrm{x}_{\mathrm{k}}\right)$ is convergent. The converse of the statement may not hold in general.

Proof. Let $x=\left(x_{k}\right)$ be a convergent sequence. Then for given $\varepsilon>0$, there exists a natural number $N$ and real or complex number $l$ such that, for every $k \geq N$, we have $\left|x_{k}-l\right|<\varepsilon$. Now, we have

$$
\begin{aligned}
\left|\Delta^{n} x_{k}\right| & =\left|\sum_{i=0}^{n}(-1)^{i}\left(\begin{array}{c}
n \\
i
\end{array}\right) x_{k+i}\right| \\
& =\left|\sum_{i=0}^{n}(-1)^{i}\left(\begin{array}{c}
n \\
i
\end{array}\right) x_{k+i}-\sum_{i=0}^{n}(-1)^{i}\left(\begin{array}{c}
n \\
i
\end{array}\right) l+\sum_{i=0}^{n}(-1)^{i}\left(\begin{array}{c}
n \\
i
\end{array}\right) l\right| \\
& =\left|\sum_{i=0}^{n}(-1)^{i}\left(\begin{array}{c}
n \\
i
\end{array}\right)\left(x_{k+i}-l\right)+l \sum_{i=0}^{n}(-1)^{i}\left(\begin{array}{c}
n \\
i
\end{array}\right)\right|
\end{aligned}
$$




$$
\begin{aligned}
& \leq \sum_{i=0}^{n}(-1)^{i}\left(\begin{array}{c}
n \\
i
\end{array}\right)\left|\left(x_{k+i}-l\right)\right|+|l|\left|\sum_{i=0}^{n}(-1)^{i}\left(\begin{array}{c}
n \\
i
\end{array}\right)\right| \\
& \leq \varepsilon \sum_{i=0}^{n}(-1)^{i}\left(\begin{array}{c}
n \\
i
\end{array}\right)=0, \text { for every } k \geq N .
\end{aligned}
$$

Therefore, $\left|\Delta^{n} x_{k}\right| \rightarrow 0$ as $k \rightarrow \infty$. For the converse part we take the following counter example:

For a natural number $m$, consider the sequence $x=\left(x_{k}\right)$, defined by $x_{k}=k^{m}$ for all $k \in \mathbb{N}_{0}$. Clearly, $x=\left(x_{k}\right)$ is divergent, but its associated difference sequence is

$$
\begin{aligned}
& \Delta^{n} x_{k}=\sum_{i=0}^{n}(-1)^{i}\left(\begin{array}{l}
n \\
i
\end{array}\right)(k+i)^{m} \\
& =k^{m}-\left(\begin{array}{c}
n \\
1
\end{array}\right)\left[k^{m}+\left(\begin{array}{c}
m \\
1
\end{array}\right) k^{m-1}+\left(\begin{array}{c}
m \\
2
\end{array}\right)+\cdots+\left(\begin{array}{l}
m \\
m
\end{array}\right)\right] \\
& +\left(\begin{array}{l}
n \\
2
\end{array}\right)\left[k^{m}+2\left(\begin{array}{c}
m \\
1
\end{array}\right) k^{m-1}+2^{2}\left(\begin{array}{c}
m \\
2
\end{array}\right)+\cdots+\left(\begin{array}{c}
m \\
m
\end{array}\right) 2^{m}\right]+\ldots \\
& +(-1)^{n}\left(\begin{array}{l}
n \\
n
\end{array}\right)\left[k^{m}+n\left(\begin{array}{c}
m \\
1
\end{array}\right) k^{m-1}+n^{2}\left(\begin{array}{c}
m \\
2
\end{array}\right)+\cdots+\left(\begin{array}{l}
m \\
m
\end{array}\right) n^{m}\right] \\
& =k^{m}\left[1-\left(\begin{array}{l}
n \\
1
\end{array}\right)+\left(\begin{array}{l}
n \\
2
\end{array}\right)-\left(\begin{array}{l}
n \\
3
\end{array}\right)+\cdots+(-1)^{n}\right] \\
& +k^{m-1}\left(\begin{array}{c}
m \\
1
\end{array}\right)\left[-\left(\begin{array}{l}
n \\
1
\end{array}\right)+2\left(\begin{array}{l}
n \\
2
\end{array}\right)-3\left(\begin{array}{l}
n \\
3
\end{array}\right)+\cdots+n(-1)^{n}\right] \\
& +k^{m-2}\left(\begin{array}{c}
m \\
2
\end{array}\right)\left[-\left(\begin{array}{l}
n \\
1
\end{array}\right)+2^{2}\left(\begin{array}{l}
n \\
2
\end{array}\right)-3^{2}\left(\begin{array}{l}
n \\
3
\end{array}\right)+\cdots+n^{2}(-1)^{n}\right]+\ldots \\
& +k^{m-m}\left(\begin{array}{l}
m \\
m
\end{array}\right)\left[-\left(\begin{array}{l}
n \\
1
\end{array}\right)+2^{m}\left(\begin{array}{l}
n \\
2
\end{array}\right)-3^{m}\left(\begin{array}{l}
n \\
3
\end{array}\right)+\cdots+n^{m}(-1)^{n}\right] \\
& = \begin{cases}0, & (n>m) \\
n !, & (n=m) . \\
\infty, & (n<m)\end{cases}
\end{aligned}
$$

Therefore, we conclude that for $n \geq m$ the difference sequence $\left(\Delta^{n}\left(k^{m}\right)\right)_{k}$ is convergent while the primary sequence $x=\left(k^{m}\right)$ is divergent. 
Theorem 2 The series defined in (3) is convergent for any proper fraction $\alpha>0$ if the sequence $x=\left(x_{k}\right)$ is convergent. The converse of the statement is true if the sequence involving infinite series

$$
\sum_{i=k}^{\infty}\left(\begin{array}{c}
i-k+\alpha-1 \\
i-k
\end{array}\right) \Delta^{\alpha}\left(x_{i}\right) \text { converges. }
$$

Proof. The proof of the sufficient part is similar to that of Theorem (1), hence omitted.

For the necessary part we assume that the difference sequence $\Delta^{\alpha} \chi_{k}$ and the infinite series $\sum_{i=k}^{\infty}\left(\begin{array}{c}i-k+\alpha-1 \\ i-k\end{array}\right) \Delta^{\alpha}\left(x_{i}\right)$ converge for all $k \in \mathbb{N}_{0}$. Let $\alpha$ be a proper fraction, i.e., $0<\alpha<1$. On simplifying (5), we obtain that

$$
\begin{aligned}
\sum_{i=k}^{\infty} & \left(\begin{array}{c}
i-k+\alpha-1 \\
i-k
\end{array}\right) \Delta^{\alpha}\left(x_{i}\right) \\
= & \left(\begin{array}{c}
\alpha-1 \\
0
\end{array}\right) \Delta^{\alpha}\left(x_{k}\right)+\left(\begin{array}{l}
\alpha \\
1
\end{array}\right) \Delta^{\alpha}\left(x_{k+1}\right)+\left(\begin{array}{c}
\alpha+1 \\
2
\end{array}\right) \Delta^{\alpha}\left(x_{k+2}\right)+\ldots \\
= & x_{k}-\left(\begin{array}{l}
\alpha \\
1
\end{array}\right) x_{k+1}+\left(\begin{array}{l}
\alpha \\
2
\end{array}\right) x_{k+2}-\left(\begin{array}{l}
\alpha \\
3
\end{array}\right) x_{k+3}+\ldots \\
& +\left(\begin{array}{c}
\alpha \\
1
\end{array}\right)\left[x_{k+1}-\left(\begin{array}{c}
\alpha \\
1
\end{array}\right) x_{k+2}+\left(\begin{array}{l}
\alpha \\
2
\end{array}\right) x_{k+3}-\left(\begin{array}{c}
\alpha \\
3
\end{array}\right) x_{k+4}+\ldots\right] \\
& +\left(\begin{array}{c}
\alpha+1 \\
2
\end{array}\right)\left[x_{k+2}-\left(\begin{array}{c}
\alpha \\
1
\end{array}\right) x_{k+3}+\left(\begin{array}{c}
\alpha \\
2
\end{array}\right) x_{k+4}-\left(\begin{array}{c}
\alpha \\
3
\end{array}\right) x_{k+5}+\ldots\right]+\ldots \\
= & x_{k} .
\end{aligned}
$$

Thus, from the hypothesis, the sequence $\left(x_{k}\right)$ is convergent. However, from Example 5, it is noticed that for a unbounded sequence $x=\left(x_{k}\right)$ with $x_{k}=k$ for all $k \in \mathbb{N}_{0}$, for a proper fraction $\alpha$, corresponding difference sequence $\Delta^{\alpha} x_{k} \rightarrow \infty$, as $k \rightarrow \infty$. This completes the proof.

Theorem 3 The series defined in (4) is convergent for any proper $\alpha>0$ or $\alpha=\mathrm{n} \in \mathbb{N}_{0}$ if the sequence $\mathrm{x}=\left(\mathrm{x}_{\mathrm{k}}\right)$ is convergent with $\mathrm{x}_{\mathrm{k}}=\mathcal{O}\left(\mathrm{k}^{-\alpha-1}\right)$. The converse of the statement is true if the sequence involving infinite series

$$
\sum_{i=k}^{\infty}(-1)^{i-k}\left(\begin{array}{c}
\alpha \\
i-k
\end{array}\right) \Delta^{-\alpha}\left(x_{i}\right) \text { converges. }
$$

Proof. We know that the infinite series in (4) represents the inverse fractional difference sequence of the sequence $\left(x_{k}\right)$, thus it always suggests the idea analog 
to integration or summation. Since the equation is a sum of infinite terms with all positive coefficients of $x_{k}$, most of the cases it gives $\infty$ even if the primary sequence is convergent. As a result, we need to consider strictly the order of the convergence of the primary sequence $\left(x_{k}\right)$ in such a way that the final sum of the series (4) will be dominated.

Let us consider the convergent sequence $x=\left(x_{k}\right)$ with $x_{k}=\mathcal{O}\left(k^{-\alpha-1}\right)$ and $\alpha>0$. Then, there exists a constant $M$ such that

$$
\sup _{k}\left|x_{k}\right| \leq \frac{M}{k^{\alpha+1}}
$$

In fact, the above sequence is a null sequence and the corresponding inverse difference sequence is given below:

$$
\begin{aligned}
\Delta^{-\alpha} x_{k} & =\sum_{i=0}^{\infty} \frac{\alpha(\alpha+1) \ldots(\alpha+i-1)}{\Gamma(i+1)} x_{k+i} \\
& =x_{k}+\alpha x_{k+1}+\frac{\alpha(\alpha+1)}{2 !} x_{k+2}+\frac{\alpha(\alpha+1)(\alpha+1)}{3 !} x_{k+3}+\ldots \\
& \leq \frac{M}{k^{\alpha+1}}\left(1+\alpha+\frac{\alpha(\alpha+1)}{2 !}+\frac{\alpha(\alpha+1)(\alpha+1)}{3 !}+\ldots\right) .
\end{aligned}
$$

The right hand side of the above equation is tending to 0 as $k \rightarrow \infty$. The equation contains two terms out of which the term $\frac{M}{k^{\alpha+1}}$ is dominating since it contains $(\alpha+1)$ as power of $1 / k$ whereas other term contains $\alpha$, only, which is a constant. It is rapidly tending to 0 as comparison to the rate at which the other term goes to $\infty$. The converse part of this theorem is similar to that of Theorem 5 .

Theorem 4 Let $\alpha>0$ be either a fraction or a natural number and $\Delta^{\alpha}: w \rightarrow$ $w$ is a linear operator provided the series in (3) is convergent.

Theorems (1), (2) and (3) can be verified in the light of the above theorem, it can be shown that most of the results are not satisfied in general.

Theorem 5 For any proper fractions $\alpha, \alpha_{1}$ and $\alpha_{2}$, in general we have

(i) $\Delta^{\alpha_{1}}\left(\Delta^{\alpha_{2}} x_{k}\right) \neq \Delta^{\alpha_{1}+\alpha_{2}}\left(x_{k}\right)$ and $\Delta^{\alpha_{2}}\left(\Delta^{\alpha_{1}} x_{k}\right) \neq \Delta^{\alpha_{1}+\alpha_{2}}\left(x_{k}\right)$,

(ii) $\Delta^{\alpha}\left(\Delta^{-\alpha} x_{k}\right) \neq x_{k}$ and $\Delta^{-\alpha}\left(\Delta^{\alpha} x_{k}\right) \neq x_{k}$,

Proof. We prove theorem by using suitable counter examples. 
Example 5 Consider the sequence $\mathrm{x}=\left(\mathrm{x}_{\mathrm{k}}\right)$, defined by $\mathrm{x}_{\mathrm{k}}=\mathrm{k}$ for all $\mathrm{k} \in \mathbb{N}_{0}$. Clearly it is a divergent sequence. Let us take $\alpha_{1}=1 / 2=\alpha_{2}$ and therefore, $\alpha_{1}+\alpha_{2}=1$. Then, we can calculate

$$
\begin{aligned}
\Delta^{\alpha_{2}} \chi_{k}= & \left(\Delta^{1 / 2} k\right)_{k}=k-\left(\begin{array}{c}
1 / 2 \\
1
\end{array}\right)(k+1)+\left(\begin{array}{c}
1 / 2 \\
2
\end{array}\right)(k+2)-\left(\begin{array}{c}
1 / 2 \\
3
\end{array}\right)(k+3)+\ldots \\
= & k\left[1-\left(\begin{array}{c}
1 / 2 \\
1
\end{array}\right)+\left(\begin{array}{c}
1 / 2 \\
2
\end{array}\right)-\left(\begin{array}{c}
1 / 2 \\
3
\end{array}\right)+\ldots\right] \\
& -\frac{1}{2}\left[1-\left(\begin{array}{c}
-1 / 2 \\
1
\end{array}\right)+\left(\begin{array}{c}
-1 / 2 \\
2
\end{array}\right)-\left(\begin{array}{c}
-1 / 2 \\
3
\end{array}\right)+\ldots\right] \\
= & \infty .
\end{aligned}
$$

Now, $\Delta^{\alpha_{1}}\left(\Delta^{\alpha_{2}}\left(x_{k}\right)\right)=\Delta^{1 / 2}\left(\Delta^{1 / 2}(k)\right)=\Delta^{1 / 2}(\infty)=\infty$, but $\Delta^{\alpha_{1}+\alpha_{2}}\left(x_{k}\right)=$ $\Delta^{1 / 2+1 / 2}(k)=\Delta(k)=k-(k+1)=-1$. Interchanging $\alpha_{1}$ and $\alpha_{2}$ in above expression we can prove the second condition. This completes the proof of Part (i) of Theorem 5.

Example 6 Let us consider the sequence $x=\left(x_{k}\right)$, defined by $x_{k}=r$ for all $\mathrm{k} \in \mathbb{N}_{0}$ and $\mathrm{r} \in \mathbb{R}$, the set of all real numbers. Clearly, $\mathrm{x}=\left(\mathrm{x}_{\mathrm{k}}\right)$ is a convergent sequence. Taking $\alpha=1 / 2$, we have

$$
\begin{aligned}
\Delta^{-\alpha} \chi_{k} & =\left(\Delta^{-1 / 2} \mathrm{r}\right)_{k}=r\left[1-\left(\begin{array}{c}
-1 / 2 \\
1
\end{array}\right)+\left(\begin{array}{c}
-1 / 2 \\
2
\end{array}\right)-\left(\begin{array}{c}
-1 / 2 \\
3
\end{array}\right)+\ldots\right] \\
& =\infty
\end{aligned}
$$

Thus, the left hand side of 1 st equation of Part (ii) is $\Delta^{\alpha}\left(\Delta^{-\alpha}\left(x_{k}\right)\right)=\Delta^{1 / 2}\left(\Delta^{-1 / 2}\right.$ $(\mathrm{r}))=\Delta^{1 / 2}(\infty)=\infty$, whereas the right hand side is $\mathrm{x}_{\mathrm{k}}=\mathrm{r}$. Again by interchanging the positions of $\alpha$ and $-\alpha$, it is also noticed that

$$
\begin{aligned}
\Delta^{\alpha} x_{k} & =\left(\Delta^{1 / 2} r\right)_{k}=r\left[1-\left(\begin{array}{c}
1 / 2 \\
1
\end{array}\right)+\left(\begin{array}{c}
1 / 2 \\
2
\end{array}\right)-\left(\begin{array}{c}
1 / 2 \\
3
\end{array}\right)+\ldots\right] \\
& =0 .
\end{aligned}
$$

Now, the left hand side of the second equation of Part (ii) can be found as $\Delta^{-\alpha}\left(\Delta^{\alpha}\left(x_{k}\right)\right)=\Delta^{-1 / 2}\left(\Delta^{1 / 2}(r)\right)=\Delta^{-1 / 2}(0)=0$ which is not equal to the right hand side i.e., $\boldsymbol{x}_{\mathrm{k}}=\mathrm{r}$. This completes the proof of Part (ii) of Theorem 5 .

Above examples conclude that linearity and exponent rules involving the fractional difference operator $\Delta^{\alpha}$ for any sequence in $w$ are not uniformly 
posed. Eventually, these rules are deviating due to lack of convergence of related infinite series. In fact, the convergence of the related infinite series is completely depending on the nature of the primary sequence $\left(x_{k}\right)$ and the choice of the values of $\alpha$. It is understood that if the primary sequence $\left(x_{k}\right)$ and the value $\alpha$ are suitably chosen then obviously, this deviation can be restricted to a given domain. This idea suggests that Theorems 1, 2 and 3 of [4] need relevant modifications and the modified results are as follows.

Theorem 6 For any positive proper fractions $\alpha, \alpha_{1}$ and $\alpha_{2}$, we have

(i) Let the sequence $\mathrm{x}=\left(\mathrm{x}_{\mathrm{k}}\right)$ be convergent, then

$$
\Delta^{\alpha_{1}}\left(\Delta^{\alpha_{2}}\left(x_{k}\right)\right)=\Delta^{\alpha_{1}+\alpha_{2}}\left(x_{k}\right)=\Delta^{\alpha_{2}}\left(\Delta^{\alpha_{1}}\left(x_{k}\right)\right),
$$

(ii) Let the sequence $\left(\Delta^{-\alpha} \chi_{k}\right)$ be convergent, then

$$
\Delta^{\alpha}\left(\Delta^{-\alpha} x_{k}\right)=x_{k},
$$

(iii) Let the sequence $\left(\Delta^{\alpha} \chi_{\mathrm{k}}\right)$ be of $\mathcal{O}\left(\mathrm{k}^{-\alpha-1}\right)$, then

$$
\Delta^{-\alpha}\left(\Delta^{\alpha} x_{k}\right)=x_{k}
$$

Combining all points, Theorem 6 can be restated as follows:

Remark 1 Let $\alpha>0$ and $\beta$ be a real such that $\alpha+\beta>0$ and the sequence $\left(x_{k}\right)$ be of $\mathcal{O}\left(k^{-m-1}\right)$, where $\mathrm{m}=\min (|\alpha|,|\beta|)$, then

$$
\Delta^{\alpha}\left(\Delta^{\beta}\left(x_{k}\right)\right)=\Delta^{\alpha+\beta}\left(x_{k}\right)=\Delta^{\beta}\left(\Delta^{\alpha}\left(x_{k}\right)\right) .
$$

Corollary 1 For any $\mathrm{n} \in \mathbb{N}$, let $\Delta^{-n}$ be the negative integral difference operator, then

(i) $\Delta^{-1}\left(x_{k}\right)=\sum_{i=1}^{\infty} x_{k+i}$, if the sequence $\left(x_{k}\right)$ is convergent with $x_{k}=\mathcal{O}\left(k^{-2}\right)$,

(ii) $\Delta^{-2}\left(x_{k}\right)=\sum_{i=1}^{\infty}(i+1) x_{k+i}$, if the sequence $\left(x_{k}\right)$ is convergent with $x_{k}=$ $\mathcal{O}\left(k^{-3}\right)$

(iii) $\Delta^{-3}\left(x_{k}\right)=\sum_{i=1}^{\infty}\left(s_{i}\right) x_{k+i}$, where $s_{i}=\sum_{j=1}^{i} j$, if the sequence $\left(x_{k}\right)$ is convergent with $\mathrm{x}_{\mathrm{k}}=\mathcal{O}\left(\mathrm{k}^{-4}\right)$. 
To next, we discuss some operator norms involving the difference operator of fractional order.

Let $A=\left(a_{n k}\right)$ be an infinite matrix with $a_{n k} \geq 0$ for all $n, k \in \mathbb{N}_{0}$. Then we have the following theorems on operator norms via the infinite matrix $A$ :

Theorem 7 Let $\mathrm{X} \in\left\{\mathrm{c}_{0}, \mathrm{c}, \ell_{\infty}\right\}$. Then the infinite matrix $\mathrm{A}$ is a bounded operator from $\mathrm{X}$ to $\mathrm{X}\left(\Delta^{\alpha}\right)$ if

$$
\mathcal{M}=\sup _{n}\left\{\sum_{k=0}^{\infty}\left|\sum_{i=0}^{\infty} \frac{(-\alpha)(-\alpha+1) \ldots(-\alpha+i-1)}{\Gamma(i+1)} a_{n+i, k}\right|\right\}<\infty
$$

and

$$
\|A\|_{\left(\infty, \Delta^{\alpha}\right)}=\mathcal{M}
$$

Proof. Suppose $X=\ell_{\infty}$ and $x \in X$. Then, we have

$$
\begin{aligned}
\|A x\|_{\left(\infty, \Delta^{\alpha}\right)} & =\sup _{n}\left|\sum_{k=0}^{\infty} \sum_{i=0}^{\infty} \frac{(-\alpha)(-\alpha+1) \ldots(-\alpha+i-1)}{\Gamma(i+1)} a_{n+i, k} x_{k}\right| \\
& \leq \sup _{n}\left\{\sum_{k=0}^{\infty}\left|\sum_{i=0}^{\infty} \frac{(-\alpha)(-\alpha+1) \ldots(-\alpha+i-1)}{\Gamma(i+1)} a_{n+i, k} x_{k}\right|\right\} \\
& \leq \mathcal{M}\|x\|_{\infty} .
\end{aligned}
$$

Also, for $x=e=(1,1,1, \ldots)$, we have

$$
\begin{aligned}
\|A e\|_{\left(\infty, \Delta^{\alpha}\right)} & =\sup _{n}\left|\sum_{k=0}^{\infty} \sum_{i=0}^{\infty} \frac{(-\alpha)(-\alpha+1) \ldots(-\alpha+i-1)}{\Gamma(i+1)} a_{n+i, k}\right| \\
& =\sup _{n}\left\{\sum_{k=0}^{\infty}\left|\sum_{i=0}^{\infty} \frac{(-\alpha)(-\alpha+1) \ldots(-\alpha+i-1)}{\Gamma(i+1)}\right| a_{n+i, k}\right\} \\
& =\mathcal{M} .
\end{aligned}
$$

This proves the result.

Theorem 8 The infinite matrix $\mathrm{A}$ is a bounded operator from $\ell_{1}$ to $\ell_{1}\left(\Delta^{\alpha}\right)$ if

$$
\overline{\mathcal{M}}=\sup _{k}\left\{\sum_{n=0}^{\infty}\left|\sum_{i=0}^{\infty} \frac{(-\alpha)(-\alpha+1) \ldots(-\alpha+i-1)}{\Gamma(i+1)} a_{n+i, k}\right|\right\}<\infty,
$$

and

$$
\|A\|_{\left(1, \Delta^{\alpha}\right)}=\overline{\mathcal{M}}
$$


Proof. Suppose that $x \in \ell_{1}$ and $A$ be an infinite matrix, then

$$
\begin{aligned}
\|A x\|_{\left(1, \Delta^{\alpha}\right)} & =\sum_{n=0}^{\infty}\left|\sum_{k=0}^{\infty} \sum_{i=0}^{\infty} \frac{(-\alpha)(-\alpha+1) \ldots(-\alpha+i-1)}{\Gamma(i+1)} a_{n+i, k} x_{k}\right| \\
& \leq \sum_{n=0}^{\infty} \sum_{k=0}^{\infty}\left|\sum_{i=0}^{\infty} \frac{(-\alpha)(-\alpha+1) \ldots(-\alpha+i-1)}{\Gamma(i+1)} a_{n+i, k} x_{k}\right| \\
& \leq \sum_{k=0}^{\infty} \sum_{n=0}^{\infty}\left|\sum_{i=0}^{\infty} \frac{(-\alpha)(-\alpha+1) \ldots(-\alpha+i-1)}{\Gamma(i+1)} a_{n+i, k}\right|\left|x_{k}\right| \\
& \leq \overline{\mathcal{M}}\|x\|_{1} .
\end{aligned}
$$

Now, for the sequence $x=e^{(m)}$ (having 1 at $m$-th place and 0 otherwise), one can get

$$
\begin{aligned}
\left\|A e^{(m)}\right\|_{\left(1, \Delta^{\alpha}\right)} & =\sum_{n=0}^{\infty}\left|\sum_{k=0}^{\infty} \sum_{i=0}^{\infty} \frac{(-\alpha)(-\alpha+1) \ldots(-\alpha+i-1)}{\Gamma(i+1)} a_{n+i, k} x_{k}\right| \\
& =\sum_{n=0}^{\infty}\left|\sum_{i=0}^{\infty} \frac{(-\alpha)(-\alpha+1) \ldots(-\alpha+i-1)}{\Gamma(i+1)} a_{n+i, m}\right| \\
& =\overline{\mathcal{M}} .
\end{aligned}
$$

This concludes the proof.

Theorem 9 The infinite matrix $\mathrm{A}$ is a bounded operator from $\ell_{\mathrm{p}},(1 \leq \mathrm{p}<\infty)$ to $\ell_{\mathrm{p}}\left(\Delta^{\alpha}\right)$ if

$$
\overline{\mathcal{M}}_{\mathfrak{p}}=\sup _{k}\left\{\sum_{n=0}^{\infty}\left|\sum_{i=0}^{\infty} \frac{(-\alpha)(-\alpha+1) \ldots(-\alpha+i-1)}{\Gamma(i+1)} a_{n+i, k}\right|^{p}\right\}<\infty,
$$

and

$$
\|A\|_{\left(p, \Delta^{\alpha}\right)}^{p}=\overline{\mathcal{M}}_{\mathrm{p}} .
$$

Proof. This follows from the proof of Theorem 8 .

Theorem 10 The identity matrix I is a bounded operator from $\mathrm{X}$ to $\mathrm{X}\left(\Delta^{\alpha}\right)$ for $\mathrm{X} \in\left\{\mathrm{c}, \mathrm{c}_{0}, \ell_{\infty}, \ell_{1}\right\}$ and

$$
\|\mathrm{I}\|_{\left(\infty, \Delta^{\alpha}\right)}=\|\mathrm{I}\|_{\left(1, \Delta^{\alpha}\right)}=2^{\alpha} .
$$


Proof. Suppose the infinite matrix $A=I$, then from Theorem 7 , we can write

$$
\begin{aligned}
\mathcal{M}_{n} & =\sum_{k=0}^{\infty}\left|\sum_{i=0}^{\infty} \frac{(-\alpha)(-\alpha+1) \ldots(-\alpha+i-1)}{\Gamma(i+1)} a_{n+i, k}\right| \\
& =\sum_{k=n}^{\infty}\left|\frac{(-\alpha)(-\alpha+1) \ldots(-\alpha+k-n-1)}{\Gamma(k-n+1)}\right| .
\end{aligned}
$$

Therefore, we have

$$
\|\mathrm{I}\|_{\left(\infty, \Delta^{\alpha}\right)}=\sup _{n} \mathcal{M}_{n}=2^{\alpha} .
$$

Similarly, using Theorem 8 , one can prove $\|\mathrm{I}\|_{\left(1, \Delta^{\alpha}\right)}=2^{\alpha}$.

\section{Conclusion}

We have investigated some idea on the convergence of difference sequence for fractional-order which may be very similar to that of integer orders but most of the cases they are nonuniform and dynamic in nature. As an application of this idea, some existing results in the literature have been modified. Certain operator norms involving the difference operator of fractional order is determined.

In the next study, we will extend this idea to the case of the statistical convergence of difference sequence and study the variations in the cases of integer and fractional orders.

\section{References}

[1] A. Alotaibi, M. Mursaleen, B. A. S. Alamri, S. A. Mohiuddine, Compact operators on some Fibonacci difference sequence spaces, J. Inequal. Appl., (2015), 2015-203.

[2] B. Altay, F. Başar, The fine spectrum and the matrix domain the difference operator $\Delta$ on the sequence space $\ell_{p},(0<p<1)$, Commun. Math. Anal., 2 (2) (2007), 1-11.

[3] C. Aydın, F. Başar, Some new difference sequence spaces, Appl. Math. Comput., 157 (3) (2004), 677-693.

[4] P. Baliarsingh, Some new difference sequence spaces of fractional order and their dual spaces, Appl. Math. Comput., 219 (18) (2013), 9737-9742. 
[5] P. Baliarsingh, S. Dutta, On the classes of fractional order difference sequence spaces and their matrix transformations, Appl. Math. Comput., 250 (2015), 665-674.

[6] P. Baliarsingh, S. Dutta, A unifying approach to the difference operators and their applications, Bol. Soc. Parana. Mat., 33 (1) (2015), 49-57.

[7] P. Baliarsingh, S. Dutta, On a spectral classification of the operator $\Delta_{v}^{r}$ over the Sequence Space $c_{0}$, Proc. Natl. Acad. Sci., India, Sect. A Phys. Sci., 84 (4) (2014) 555-561.

[8] P. Baliarsingh, On a fractional difference operator, Alexandria Eng. J., 55 (2) (2016), 1811-1816.

[9] P. Baliarsingh, L. Nayak, A note fractional difference operators, Alexandria Eng. J., 57 (2) (2018), 1051-1054.

[10] P. Baliarsingh, On certain dynamic properties of difference sequences and the fractional derivatives, Math. Metho. Appl. Sci., (2020) doi:10.1002/mma.6417.

[11] F. Başar, Summability theory and its applications, Bentham Science Publishers, e-books, Monographs, İstanbul, 2012.

[12] F. Başar, M. Kirişçi, Almost convergence and generalized difference matrix, Comput. Math. Appl., 61 (3) (2011), 602-611.

[13] F. Başar, B. Altay, On the space of sequences of p-bounded variation and related matrix map- pings, (English, Ukrainian summary) Ukrain. Mat. Zh., 55 (1) (2003), 108-118; reprinted in Ukrainian Math. J., 55 (1) (2003), 136-147.

[14] M. Basarır, E. E. Kara, On some difference sequence spaces of weighted mean and compact operators, Ann. Funct. Anal., 2 (2) (2011), 114-129.

[15] S. Dutta, P. Baliarsingh, On the fine spectra of the generalized rth difference operator $\Delta_{v}^{r}$ on the sequence space $\ell_{1}$, Appl. Math. Comput., 219 (2012), 1776-1784.

[16] S. Dutta, P. Baliarsingh, On the spectrum of 2-nd order generalized difference operator $\Delta^{2}$ over the sequence space $c_{0}$, Bol. Soc. Paran. Mat. 31 ( 2) (2013), 235-244. 
[17] M. Et, R. Çolak, On some generalized difference sequence spaces, Soochow J. Math., 21 (4) (1995), 377-386.

[18] U. Kadak, P. Baliarsingh, On certain Euler difference sequence spaces of fractional order and related dual properties, J. Nonlinear Sci. Appl., 8 (2015), 997-1004.

[19] M. Kirişçi, F. Başar, Some new sequence spaces derived by the domain of generalized difference matrix, Comput. Math. Appl., 60 (5) (2010), 1299-1309.

[20] H. Kızmaz, On Certain Sequence spaces, Canad. Math. Bull., 24 (2) (1981) 169-176.

[21] E. Malkowsky, M. Mursaleen, S. Suantai, The dual spaces of sets of difference sequences of order $\mathrm{m}$ and matrix transformations, Acta Math. Sin. (Engl. Ser.), 23 (3) (2007), 521-532.

[22] S. A. Mohiuddine, B. Hazarika, Some classes of ideal convergent sequences and generalized difference matrix operator, Filomat, 31 (6) (2017), 18271834 .

[23] M. Mursaleen, A. K. Noman, On some new difference sequence spaces of non-absolute type, Math. Comput. Modelling, 52 (2010), 603-617.

[24] M. Mursaleen, A. K. Noman, Compactness of matrix operators on some new difference sequence spaces, Linear Algebra Appl., 436 (1) (2012), $41-52$.

[25] M. Mursaleen, V. Karakaya, H. Polat, N. Simsek, Measure of noncompactness of matrix operators on some difference sequence spaces of weighted means, Comput. Math. Appl., 62 (2011), 814-820.

[26] B. C. Tripathy, On a New class of sequences, Demonstratio Math., 37 (2) (2004), 377-381.

[27] B. C. Tripathy, S. Mahanta, On a class of difference sequences related to the $\ell^{p}$ space defined by Orlicz functions, Math. Slovaca, 57 (2) (2007), $171-178$.

[28] B. C. Tripathy, A. Paul, The spectrum of the operator $D(r, 0, s, 0, t)$ over the sequence spaces $c_{0}$ and c, Journal Math., 2013, (2013), Article ID 430965 . 
[29] B. C. Tripathy, A. Paul, The spectrum of the operator D $(r, 0,0, s)$ over the sequence space $\boldsymbol{c}_{0}$ and c, Kyungpook Math. Journal, 53 (2) (2013), $247-256$.

[30] B. C. Tripathy, A. Paul, The spectrum of the operator $\mathrm{D}(\mathrm{r}, 0,0, \mathrm{~s})$ over the sequence spaces $\ell_{p}$ and $b v_{p}$, Hacettepe J. Math. Stat., 43 (3) (2014), 425-434.

Received: March 5, 2020 